\title{
Joint Discussion 14 Modeling dense stellar systems
}

\author{
Alison I. Sills ${ }^{1}$, Ladislav Subr ${ }^{2}$, and Simon F. Portegies Zwart ${ }^{3}$ (eds.) \\ ${ }^{1}$ Department of Physics and Astronomy, McMaster University, Hamilton, Canada \\ email: asills@mcmaster.ca \\ ${ }^{2}$ Astronomical Institute, Charles University, Praha 8, Czech Republic \\ email: subr@sirrah.troja.mff.cuni.cz \\ ${ }^{3}$ Astronomical Institute Anton Pannekoek, University of Amsterdam, the Netherlands \\ Preface \\ email: spz@science.uva.nl
}

Joint Discussion 14 was held at the General Assembly of the International Astronomical Union from August 17 until 23 in the beautiful Bohemian capital, Prague. The blueprints for this meeting were laid out during the MODEST-5 workshop, held in the Canadian city of Hamilton, Ontario in August 2004. We were sitting in a nice cafe with local brew and food, discussing the future of the MODEST community when we posed the idea for this Joint Discussion at the General Assembly. The meeting was then coined MODEST-7.

MODEST stands for MOdeling DEnse STellar systems, which is an initiative between scientists with a common interest in, well..., you guess. One interesting aspect of the MODEST community is that it brings together scientists who feel that the best way to make progress in their respective sub-domains in computational astrophysics and observational astronomy is by collaboration with scientists in neighboring disciplines. By close collaboration, exchanging information via workshops, conferences and via the MODEST website (<http://www.manybody.org $>$ ) great progrees has been made recently on the understanding and modeling of dense stellar systems like young star formation regions, massive star clusters, galactic star clusters, globular clusters and the nuclei of galaxies.

One of the interesting aspects of MODEST is that it brings together friend and foe, collaborator and competitor in a friendly environment. Luckily the MODEST community consists of people who do not fear competition and therefore science is discussed in the lecture room in the heat of the moment. The scientific output from this 'gang of scientists' is measured in tens of papers for which the basis has been laid at some MODEST event, most likely while appreciating some local gastronomic speciality.

In this proceedings you will find many examples of the recent progress in this open scientific community, including the summary of Douglas Heggie. For the meeting, and the open way the MODEST community operates I would like to introduce the term 'open science', to emphasis the openness in which we communicate, compete and share our findings. I hope that we will have many more occasions in which we can enjoy this open scientific community.

\section{Scientific Organizing Committee}

Christian M. Boily (France), Melvyn B. Davies (Sweden), Douglas C. Heggie (UK), Piet Hut (USA), Ralf Klessen (Germany), Junichiro Makino (Japan), Rosemary A. Mardling (Australia), Steve L. W. McMillan (USA), Georges Meylan (Switzerland), Giampaolo Piotto (Italy), Simon F. Portegies Zwart (the Netherlands), Alison I. Sills (Canada, cochair), Rainer Spurzem (Germany), and Ladislav Subr (Czech Republic, co-chair).

Simon F. Portegies Zwart, for the SOC, Amsterdam, 26 October 2006 